Théologiques

Théologiques

\title{
Modernités concurrentes
}

\section{Le sens à donner au « bien commun " dans la rencontre islamo-chrétienne}

\section{Kornel Zathureczky}

Volume 19, numéro 2, 2011

Le dialogue islamo-chrétien

URI : https://id.erudit.org/iderudit/1024728ar

DOI : https://doi.org/10.7202/1024728ar

Aller au sommaire du numéro

\section{Éditeur(s)}

Faculté de théologie et de sciences des religions, Université de Montréal

ISSN

1188-7109 (imprimé)

1492-1413 (numérique)

Découvrir la revue

Citer cet article

Zathureczky, K. (2011). Modernités concurrentes : le sens à donner au « bien commun » dans la rencontre islamo-chrétienne. Théologiques, 19(2), 65-87. https://doi.org/10.7202/1024728ar

\section{Résumé de l'article}

Cet article propose une évaluation critique des deux réponses d'envergure à "A Common Word » originaire de centres traditionnels de la chrétienté européenne. Dans les deux cas, la notion de bien commun joue un rôle central dans la détermination de la forme et de l'orientation de la coopération interreligieuse dans un contexte mondial. Alors que les réponses émanant du Vatican sont lues comme une expression de la pragmatique interreligieuse, la seconde, un engagement plus solide de Rowan Williams est présenté comme une tentative de développer une éthique interreligieuse de la communication. L'examen critique de ces deux visions de la solidarité interreligieuse conduit à des conclusions significatives quant à la notion d'autrui, une catégorie centrale partagée par toutes les traditions abrahamiques. 


\title{
Modernités concurrentes
}

\section{Le sens à donner $\mathrm{au}$ «bien commun» dans la rencontre islamo-chrétienne*}

\author{
Kornel ZATHURECZKY** \\ Sciences des religions \\ Université de Sudbury (Ontario, Canada)
}

L'un des documents religieux les plus significatifs des dernières années, (2008) «A Common Word» (CW) a été signé par un très large éventail de clercs musulmans et de chefs religieux et il s'adressait à un spectre tout aussi large de leaders chrétiens ${ }^{1}$. Le document suggère que le double devoir religieux d'amour de Dieu et d'amour du prochain interpelle autant la tradition musulmane que la tradition chrétienne à œuvrer activement à l'édification d'une paix mutuelle.

CW avance que la propension monothéiste d'adorer un seul et unique Dieu couplée au devoir d'agir envers autrui avec justice et équité sont des principes inséparables et suffisamment forts pour contribuer à rapprocher deux traditions qui ont connu trop de discorde et d'inimitié. Bien que $\mathrm{CW}$ et les réactions chrétiennes subséquentes ne proposent pas de solutions immédiates et faciles à des siècles d'hostilité, de malveillance et de calomnies théologiques entre christianisme et islam, ils représentent néanmoins le travail nécessaire, constructif et inévitablement théologique qui contribuera à jeter les bases d'une coexistence pacifique fondée sur les conceptions et les pratiques chrétiennes et musulmanes de l'amour.

* Traduit par Jacques-André Houle.

* Kornel Zathureczky est professeur adjoint en science des religions à l'Université de Sudbury. Ses recherches actuelles portent notamment sur les théories et les méthodes de l'étude scientifique des religions, les droits des minorités religieuses et la force de la loi dans l'enseignement public de la religion (Québec et Ontario). Il a récemment publié (2009) The Messianic Disruption of Trinitarian Theology, Lexington Books/ Rowman et Littlefield.

1. Pour le texte intégral original en arabe, voir le site internet officiel de CW. On y retrouve une documentation exhaustive et à jour des réactions au texte et des initiatives qui en découlent: <http://acommonword.com/>.

(C) Revue Théologiques 2011. Tout droit réservé. 
Tout en reconnaissant l'importance d'une réponse presque immédiate et largement publicisée à $\mathrm{CW}$ par des milieux théologiques progressistes aux États-Unis, le présent article s'attardera sur des réactions émanant d'Europe, le centre traditionnel du christianisme occidental, qui a historiquement été le plus touché par la présence de l'islam et de la civilisation islamique ${ }^{2}$. En choisissant ce point de vue, le présent article ne suggère aucunement quelque adhésion implicite à des théories de choc des civilisations entre l'Occident, historiquement marqué par le christianisme, et le monde musulman ${ }^{3}$. Et pourtant, il semble évident que de mettre entre parenthèses l'hyper-activisme interreligieux nord-américain afin de concentrer notre attention sur des voix issues des centres historiques du christianisme européen permet de fournir une matrice particulièrement utile à l'examen d'autres points de vue sur les tangentes des relations islamochrétiennes soulevés par la publication de CW.

L'étude qui suit aborde CW comme l'expression d'un effort concerté émanant de la communauté musulmane pour articuler une vision islamique de la place de la religion et de la coexistence interreligieuse au sein de la modernité. Afin d'arriver à appréhender correctement $\mathrm{CW}$ comme l'expression d'un islam apprivoisant lui-même la modernité, le document sera d'abord examiné en juxtaposition avec Nostra Aetate, dans lequel l'Église catholique romaine tente d'articuler sa propre place dans un environnement religieusement pluraliste profondément influencé par la modernité occidentale. L'article poursuit en examinant des réponses concrètes au défi posé par $\mathrm{CW}$ au christianisme, fortement déterminé par la donne de la modernité européenne. Nous ferons dans ce qui suit la distinction entre deux types de réponses, chacune étudiée plus en profondeur. La première constellation de réponses exprimées par des sources officielles du Vatican tente de définir le dialogue interreligieux dans la perspective d'une modernité unique, européenne. La deuxième réponse à cette invitation des musulmans lancée aux leaders chrétiens, émanant du chef de l'Église d'Angleterre et plus approfondie en matière théologique, est présentée comme une

2. La réaction la plus médiatisée est venue de la Divinity School de l'Université Yale, qui organisa également une importante conférence sur le campus de l'Université Yale. Au sujet de cette "Yale Response », qui recueillit plus de 300 signatures, voir: «Loving God and Neighbor Together: A Christian Response to A Common Word Between Us and You» in: New York Times, 11 novembre 2007.

3. Même le grand public est aujourd'hui au courant de la théorie de Samuel Huntington quant au "choc des civilisations" (Huntington 1996). Il est révélateur que cette expression ait été utilisée pour la première fois par Bernard Lewis (1990). 
volonté de replacer le dialogue dans une perspective qui reconnaît l'existence d'une modernité distincte représentée par CW. Le présent article avance que ces deux réponses représentent deux conceptions divergentes d'une théologie politique chrétienne, l'une quérulente au point d'être antagonique, l'autre polémique mais pluraliste.

\section{Le contexte de «A Common Word»: des modernités concurrentes}

Se concentrer sur les réponses européennes à CW est loin d'être un choix arbitraire. Ce choix repose largement sur le rapport étroit entre le document musulman et une allocution prononcée en 2007 par le pape Benoît XVI à l'Université de Ratisbonne en Allemagne ${ }^{4}$. Dans ce discours, le pape a semblé ressusciter une polémique du Moyen-Âge entre le christianisme et l'islam, en rapportant une citation d'un empereur byzantin, Manuel II Paléologue, au sujet des actions violentes du prophète de l'islam ${ }^{5}$. Bien que l'objectif du discours du pape Benoît ait été de présenter des arguments à l'appui du rôle pérenne de la chrétienté dans une complémentarité à la tradition philosophique grecque - une fusion décisive accomplie en Europe -, la représentation qu'il y a faite de l'islam et sa référence douteuse au Prophète n'ont pas été favorablement perçues dans les cercles islamiques ${ }^{6}$.

En réponse au discours du pape à Ratisbonne, un groupe de 38 savants musulmans ont envoyé une lettre au Vatican dans laquelle ils s'opposaient à sa représentation de l'islam et au fait qu'il laissait entendre que la conception islamique de Dieu avait pour résultat une propension inhérente à une violence légitimée par la religion (Open Letter to His Holiness). À la lumière de l'absence de réponse du Vatican, un groupe plus diversifié et

4. Pour le texte intégral de ce discours, voir le site internet du Saint-Siège: <http://www. vatican.va/holy_father/benedict_xvi/speeches/2006/september/documents/hf_benxvi_spe_20060912_university-regensburg_en.html>, version anglaise consultée par l'auteur le 15 novembre 2010; ou bien <http://www.vatican.va/holy_father/benedict_xvi/speeches/2006/september/documents/hf_ben-xvi_spe_20060912_universityregensburg_fr.html>, version française consultée par le traducteur le 11 septembre 2013. Il est important de noter que le texte original a été modifié après la réception extrêmement négative de ce discours dans le monde musulman.

5. La référence à Mahomet, que le pape caractérise comme inacceptable dans l'édition finale du discours, est la suivante: «Montre[-]moi ce que Mahomet a apporté de nouveau et tu ne trouveras que du mauvais et de l'inhumain comme ceci, qu'il a prescrit de répandre par l'épée la foi qu'il prêchait.»

6. Tariq Ramadan (2006) a offert une réaction très médiatisée et assez mesurée à ce discours. 
plus nombreux a rédigé $\mathrm{CW}$, où la place centrale qu'occupe l'amour dans les traditions abrahamiques a été proposée comme terrain d'entente sur lequel établir une coexistence et une coopération pacifiques entre christianisme et islam.

Bien que le Vatican et le pape Benoît lui-même aient nié toute intention de dépeindre l'islam de manière maligne dans le discours de Ratisbonne, suggérant même que ce qui y avait transpiré était une véritable invitation au dialogue, l'avis général des personnes bien au fait des circonstances pointe vers une perception beaucoup moins favorable du discours eurocentrique du pape.

Malgré le rôle joué par le discours de Ratisbonne pour aider à contextualiser la création de $\mathrm{CW}$, on ne peut pas considérer la publication de $\mathrm{CW}$ comme une simple une réaction à une apparente provocation. Plusieurs études situent les origines de CW comme étant l'expression d'une plus large initiative intra-musulmane (Speelman 2010). D'après cette interprétation, le dialogue intra-musulman, qui a précédé le discours de Ratisbonne, était d'une qualité telle qu'il a fini par avoir des répercussions extra-musulmanes, comme il en ressort dans l'initiative interconfessionnelle qu'est le CW. Le mouvement d'exploration interconfessionnelle entre la chrétienté et le monde musulman, déclenché par la publication de CW, avait connu des précédents dans des démarches intra-musulmanes cherchant à établir des paramètres fondamentaux quant à la représentation de l'islam dans un contexte contemporain de mondialisation.

Le principal et, finalement, le véritable événement déclencheur de CW a été la conférence d'Amman intitulée "The International Islamic Conference: True Islam and Its Role in Modern Society» (2005), organisée par le Royal Aal al-Bayt Institute for Islamic Thought de Jordanie sous le patronage du roi Abdullah II (Lumbard 2009). Cette conférence historique a réuni une grande diversité de délégués musulmans issus des sphères légale et théologique afin de se pencher sur les défis pressants de la communauté musulmane mondiale. La résolution commune d'interpeller de manière inclusive et pluraliste l'ensemble du monde musulman a eu des répercussions telles, qu'elle s'est ultimement élargie en une prise d'engagement active et significative auprès de la tradition chrétienne ${ }^{7}$.

7. Pour le mot de la fin lors de la conférence d'Amman, voir le site internet officiel du Amman Message: <http://ammanmessage.com/index.php ?option=com_content\&ta sk=view\&id=20\&Itemid=34>, consulté le 15 novembre 2010. 
Reconnaître en CW une initiative interconfessionnelle, issue d'un dialogue intra-confessionnel au sein de la communauté musulmane, comporte la valeur heuristique ajoutée d'arriver à identifier des correspondances substantielles entre les efforts de renouveau au sein des traditions musulmane et catholique romaine ${ }^{8}$. Les réalisations historiques de la conférence d'Amman, envisagées comme le résultat des efforts de la communauté musulmane mondiale pour relever les défis de la modernité, peuvent se comparer au concile Vatican II. Comme la conférence d'Amman et les buts élevés qu'elle s'était fixés, le concile a illustré les efforts de la communauté catholique romaine du monde entier pour se renouveler face au monde moderne. Malgré les énormes différences historiques et institutionnelles entre l'Église catholique romaine et l'islam, on pourrait considérer ces deux événements d'importance mondiale comme des tentatives majeures de la part des deux traditions pour confronter les défis posés par la modernité. Plus significatif encore pour les besoins de la présente étude, le ferment que renferment ces deux événements éloignés dans le temps trouve à s'exprimer à travers une approche novatrice et positive envers l'altérité religieuse. En jetant un coup d'œil rapide à la réponse catholique à la modernité qui est inscrite dans les changements amorcés par Vatican II, on arrivera à élargir davantage le contexte qui permettra d'évaluer les réponses européennes à CW.

Parmi les documents du concile Vatican II, il y en a un assez bref qui retient l'attention en ce qu'il révèle un changement important dans l'évaluation que fait l'Église catholique des autres traditions religieuses. Dans le document Nostra Aetate, déclaration sur les relations de l'Église avec les religions non chrétiennes, on trouve une importante section consacrée à l'islam affirmant explicitement la reconnaissance de la part de l'Église de la communauté universelle musulmane, de même que l'importance d'aller à la rencontre de cette communauté sur la base des efforts de l'Église de construire un avenir de justice et de paix pour l'humanité.

Il vaut la peine ici de citer la section de Nostra Aetate qui rapporte l'opinion de l'Église sur la communauté musulmane et ses croyances:

L'Église regarde aussi avec estime les musulmans, qui adorent le Dieu unique, vivant et subsistant, miséricordieux et tout-puissant, créateur du ciel

8. Une excellente source théorique est Armando Salvatore (2007). Voir aussi Baum (2009). Une importante initiative internationale vouée à l'exploration de liens entre le catholicisme et l'islam a été lancée par le Kroc Institute for International Peace Studies de l'Université Notre-Dame, sous l'égide de «Contending Modernities », voir: <http:// kroc.nd.edu/research/religion-conflict-peacebuilding/contending-modernities>. 
et de la terre, qui a parlé aux hommes. Ils cherchent à se soumettre de toute leur âme aux décrets de Dieu, même s'ils sont cachés, comme s'est soumis à Dieu Abraham, auquel la foi islamique se réfère volontiers. Bien qu'ils ne reconnaissent pas Jésus comme Dieu, ils le vénèrent comme prophète; ils honorent sa Mère virginale, Marie, et parfois même l'invoquent avec piété. De plus, ils attendent le jour du jugement, où Dieu rétribuera tous les hommes après les avoir ressuscités. Aussi ont-ils en estime la vie morale et rendent-ils un culte à Dieu, surtout par la prière, l'aumône et le jeûne. [...] Même si, au cours des siècles, de nombreuses dissensions et inimitiés se sont manifestées entre les chrétiens et les musulmans, le saint Concile les exhorte tous à oublier le passé et à s'efforcer sincèrement à la compréhension mutuelle, ainsi qu'à protéger et à promouvoir ensemble, pour tous les hommes, la justice sociale, les valeurs morales, la paix et la libertée

Dans son stimulant compte-rendu de CW, Daniel Madigan, s.j., de la Commission pontificale pour les rapports religieux avec les musulmans, fait remarquer que Nostra Aetate continue d'être considérée comme la position officielle de l'Église catholique sur les rapports avec la communauté musulmane (Madigan 2008). De plus, il propose qu'une manière possible de comprendre la réception de $\mathrm{CW}$ par les catholiques romains est de reconnaître dans celui-ci une sorte de réponse aux affirmations de Nostra Aetate concernant les musulmans. À la lumière de cela, CW peut être vu comme un document qui reconnaît tacitement l'initiative de Nostra Aetate tout en offrant une élaboration des raisons musulmanes pour favoriser la poursuite des rapprochements entre les deux traditions.

Tout en évoquant l'importance initiale de Nostra Aetate pour le dialogue entre chrétiens et musulmans, Madigan indique un important déplacement de l'axe du dialogue interreligieux: jusqu'ici largement positionné du côté chrétien, celui-ci se déplace désormais du côté de la communauté musulmane internationale. Madigan note deux caractéristiques importantes de ce changement. La première est le fléchissement de l'enthousiasme catholique quant au dialogue interreligieux ${ }^{10}$. Le déclin palpable des initia-

9. Voir le site internet du Saint-Siège: <http://www.vatican.va/archive/hist_councils/ ii_vatican_council/documents/vat-ii_decl_19651028_nostra-aetate_fr.html>, consulté le 11 septembre 2013.

10. On se doit de noter un changement notable dans l'approche de l'Église catholique quant au dialogue interreligieux, avec la Déclaration «Dominus Iesus », sur l'unicité et l'universalité salvifique de Jésus-Christ et de l'Église, publiée par la Congrégation pour la doctrine de la foi dirigée par le cardinal Joseph Ratzinger. Voir: http://www. vatican.va/roman_curia/congregations/cfaith/documents/rc_con_cfaith_doc_2000 0806_dominus-iesus_en.html, consultée par l'auteur le 15 novembre 2010. http:// 
tives interreligieuses peut être vu comme le résultat de la dynamique assez univoque du dialogue immédiatement après les déclarations du concile. Un autre développement en ce qui concerne le déplacement de l'épicentre du dialogue religieux est en lien avec l'intensification du dialogue intra-musulman, comme en témoigne $\mathrm{CW}$. Ce document - et il y a ici des correspondances claires avec l'impact de Nostra Aetate - a une importance pédagogique non équivoque en ce qu'il appelle la communauté musulmane dans son ensemble à résister aux fausses représentations de la communauté islamique, qu'elles viennent de l'intérieur ou de l'extérieur. Depuis la promulgation de Nostra Aetate, l'islam est devenu une réalité européenne. La mobilisation à l'intérieur de la communauté musulmane survient à un moment clé où il y a une tendance dans les cercles catholiques au repli sur soi sans chercher à résister à l'émergence alarmante de sentiments exprimés dans le discours politique européen propageant des idées dégradantes et simplistes sur le compte de la population musulmane d'Europe.

À la lumière des changements affectant la démographie religieuse du continent européen, l'appel au dialogue interreligieux lancé par $\mathrm{CW}$ sert à rappeler aux catholiques d'Europe qu'ils doivent prendre au sérieux leurs responsabilités envers leurs concitoyens musulmans. La responsabilité de trouver un accord mutuel, clairement présentée par Nostra Aetate quant aux attitudes que doivent adopter les catholiques envers les musulmans, est devenue une responsabilité politique enjoignant l'Église à confronter les puissances politiques qui instrumentalisent les peurs injustifiées de l'électorat. Ici, Madigan rappelle les ramifications politiques de l'appel à revenir au cœur monothéiste qui unit les trois religions abrahamiques. La nature englobante de l'appel à se dévouer entièrement à Dieu suppose l'impossibilité de prêter allégeance à un projet politique ou à une idéologie politique, surtout quand cette dernière tente d'utiliser la religion comme une source de sa légitimation.

\section{Des réponses d'Europe}

Après avoir soulevé les parallèles entre Nostra Aetate et CW et le déplacement de l'essentiel des initiatives interconfessionnelles des catholiques vers les musulmans, on peut faire la distinction entre deux types de réponses à

www.vatican.va/roman_curia/congregations/cfaith/documents/rc_con_cfaith_ doc_20000806_dominus-iesus_fr.html, version française consultée par le traducteur le 11 septembre 2013. 
CW émanant des centres traditionnels de la chrétienté européenne. Le premier type de réponse cherche à engager l'initiative interconfessionnelle musulmane en la réduisant à sa portée pragmatique. Cette approche est essentiellement celle de la position officielle du Vatican. Un autre type de réponse - et ici l'engagement de Rowan Williams, archevêque de Cantorbéry, en est l'exemple le plus notable - cherche à prendre CW au pied de la lettre, comme une invitation au dialogue interconfessionnel entre les traditions; ainsi, cette approche est caractérisée par une orientation théologique d'envergure.

Dans ce qui suit, nous examinerons d'abord des réponses qui sont représentatives de celles émanant du Vatican. Après un aperçu des réponses vaticanes les plus importantes à ce jour, nous porterons notre attention sur la réponse de l'archevêque de Cantorbéry, un engagement qui repose sur de solides bases théologiques.

\subsection{Les réponses du Vatican: un pragmatisme interreligieux}

Après une période de réticence suivant la publication de CW, le Vatican a expédié une lettre en 2007 qui constituait la première réaction officielle à $\mathrm{CW}^{11}$. La lecture de cette lettre nous apprend que le Vatican a interprété $\mathrm{CW}$ comme un geste positif, y reconnaissant une contribution importante aux efforts communs visant à promouvoir la paix dans le monde. La lettre du Vatican insistait sur le fait qu'en dépit des différences évidentes qui divisent les musulmans et les chrétiens, il existe des croyances fondamentales que partagent les deux religions, en particulier la foi en un seul Dieu et l'anticipation du Jugement dernier. À cet égard, la lettre ne faisait que reprendre ce qui avait déjà été exprimé dans Nostra Aetate.

La lettre permettait également d'apprécier la réaction du pape Benoît à l'accent mis dans le document sur le double commandement, dans lequel il percevait une résonnance au thème central de sa lettre encyclique Deus caritas est. Dans cette encyclique, le pape Benoît approfondit la place centrale qu'occupe l'amour dans la tradition chrétienne et soutien que la conception chrétienne de l'amour est un élément important pour tenter de

11. Voir la lettre du Vatican au prince Ghazi, datée du 19 novembre 2007, à <http:// acommonword.com/lib/ downloads/letter-from-the-vatican.pdf >, consulté le 15 novembre 2010. 
relever les défis éthiques complexes d'une société moderne, sécularisée et mondialisée ${ }^{12}$.

Reprenant le thème de l'amour de l'encyclique, l'archevêque Celestino Migliore, nonce apostolique et observateur permanent du Saint-Siège près de l'Organisation des Nations Unies, appelle à lutter contre les profonds préjugés et la méfiance qui empoisonnent les relations entre musulmans et chrétiens (Migliore 2008). Migliore remarque que les deux communautés ont construit et fonctionnent souvent avec des images monstrueuses projetées sur l'autre, le lointain étranger. Le rapprochement est un geste crucial qui permet aux deux traditions de dissiper les images déformées de l'autre et d'arriver à reconnaître dans la présence de l'autre celle d'un frère vivant à proximité. Migliore suggère que pour qu'il y ait une rencontre significative et une coopération avec son frère musulman, il faut qu'il y ait reconnaissance de points de divergence parmi les points de convergence avec la tradition islamique. Alors que les principaux points de contact entre christianisme et islam - soit leur commune généalogie abrahamique, leur propension monothéiste partagée et la place fondamentale d'un texte révélé - offrent des possibilités importantes de partage et de collaboration, Migliore soutient que la reconnaissance de différences dans ces trois aspects est déterminante afin de chasser les tendances historiquement enracinées à projeter sur l'autre des images monstrueuses. Cette voie équilibrée pour promouvoir un rapprochement entre deux traditions prépare le terrain "pour atteindre ensemble la sainteté personnelle et le bien commun de la société » (Migliore 2008, 3).

Bien que chacune de ces conséquences d'une rencontre significative entre musulmans et chrétiens s'avère essentielle à l'édification de la paix dans une communauté mondiale déchirée par la division et la violence, la condition préalable primordiale à la construction de la paix dans le monde, selon Migliore, ne repose pas uniquement sur le dialogue interreligieux. Plutôt, s'inspirant des préoccupations singulièrement européennes du pape Benoît XVI, la coexistence pacifique dans le cadre complexe d'une société mondialisée doit s'appuyer sur la réconciliation entre foi et raison. Cette réconciliation est non seulement le remède aux pathologies de la raison et de la foi, mais est également la condition préalable à une véritable manifestation de la dignité de la personne. C'est sur cet horizon d'une anthropologie

12. Voir l'encyclique papale Deus caritas est à <http://www.vatican.va/holy_father/ benedict_xvi/encyclicals/ documents/hf_ben-xvi_enc_20051225_deus-caritas-est_ fr.html>. 
reposant sur l'harmonie entre foi et raison que Migliore situe l'importance de CW. La proposition lancée par CW d'unir chrétiens et musulmans sous le signe du double commandement d'amour de Dieu et d'amour du prochain est vue comme "le fondement théologique le plus solide" pour la coexistence pacifique et la reconnaissance mutuelle entre musulmans et chrétiens dans la communauté mondiale:

De fait, si chrétiens et musulmans redécouvrent ensemble la profondeur et la fécondité du message que leurs textes respectifs livrent au sujet de l'amour de Dieu et du prochain - un prochain qui désigne l'humanité dans toute sa diversité, sans exception ni discrimination - , nous contribuerons alors à répandre la lumière et la bonne volonté afin de résoudre de nombreuses questions sur le respect mutuel et la collaboration pour la paix. (Migliore 2008, 6)

Migliore souligne les ramifications universelles du commandement divin d'amour de Dieu et du prochain, qui dépassent les confins du dialogue chrétiens-musulmans pour atteindre le domaine plus vaste des droits universels de la personne. En tant que représentant du Vatican auprès de l'ONU, Migliore propose une perspective qui situe CW sur l'horizon de la communauté politique mondiale des nations. Il mentionne d'importantes initiatives de l'ONU pour favoriser le dialogue entre civilisations (par exemple L'Alliance des civilisations). L'apport de Migliore à la réaction catholique à CW est d'avoir su étoffer un pragmatisme significatif axé sur l'atteinte de la paix. Faisant référence à un important ouvrage de Raymond Helmick, s.j., Migliore souligne les contributions uniques et les impératifs particuliers auxquels certaines traditions font face en puisant sur la spécificité même de leurs propres ressources pour gérer les conflits (Helmick 2001). Le point de départ du dialogue dépend en fin de compte de la reconnaissance pleine et authentique de la valeur et de la dignité de l'individu, sans égard à son affiliation religieuse. Seule cette reconnaissance fondamentale a le pouvoir d'oblitérer des consciences l'image monstrueuse de l'autre. Migliore admet que les dialogues initiés par l'ONU ont des limites et qu'une rencontre des civilisations dépend de la coopération sincère et respectueuse de ceux qui témoignent de leur foi et de leur dévotion à Dieu par l'établissement de ponts entre membres de diverses confessions religieuses. D'où l'importance de poursuivre le dialogue interreligieux.

Comme mentionné plus haut, l'Église catholique romaine a officiellement reconnu, dans Nostra Aetate, à la fois l'importance de reconnaître la présence de l'Esprit universel dans les traditions religieuses non chrétiennes 
et le besoin spirituel de l'Église d'engager un dialogue avec d'autres traditions religieuses. Afin de réaliser le mandat élargi du dialogue interreligieux tel que conçu par Vatican II, l'Église a institué en 1964 le Conseil pontifical pour le dialogue interreligieux. Suite à la publication de CW, le président du Conseil pontifical, le cardinal Jean-Louis Tauran, a offert quelques pistes de réflexion sur la dynamique du dialogue interreligieux, avec une attention toute particulière à l'engagement de l'Église auprès des musulmans (Cardinal Tauran 2008) ${ }^{13}$. Sur la question capitale du sens à donner et du but ultime du dialogue interreligieux, l'avis de Tauran renvoie à la surprenante appropriation du personnalisme exprimée dans le discours de Ratisbonne du pape Benoît XVI. La coexistence harmonieuse chez l'homme de la foi et de la raison définit les limites de l'orientation interrogative et dialogique du Soi. Le dialogue avec Soi se prolonge par un dialogue avec l'autre, dont le but est l'intercompréhension. C'est cette intercompréhension qui amène toujours à prendre des risques, démarche qui est au cœur du dialogue interreligieux. Le dialogue entre traditions religieuses prend plusieurs visages: c'est un dialogue de vie, un dialogue d'œuvres, un dialogue théologique et finalement un dialogue de spiritualités. Le dialogue interreligieux représente en définitive un bien commun pour la communauté mondiale. Tauran voit en CW une initiative importante, qui reconnaît qu'il est de la responsabilité de tous de travailler pour le bien commun et de manière concertée pour la paix.

Les réactions à $\mathrm{CW}$ rapportées plus haut sont typiques d'une sorte d'engagement qui s'appuie sur des impératifs pratiques pour dicter la coopération et la solidarité entre deux religions. Cette optique est confirmée lorsqu'on examine l'allocution du pape Benoît au Forum catholiquemusulman, une tribune convoquée pour faire suite à $\mathrm{CW}^{14}$. À la lecture du discours du pape, il devient évident qu'en dépit de sa perspicacité théologique, il évite de s'engager dans quelque discussion théologique de fond quant à l'invitation de CW d'examiner les points en commun des deux traditions. Dans son mot de la fin adressé à un éminent groupe de délégués

13. Texte d'abord présenté sous forme de discours au Heythrop College (2008). Aussi, le cardinal Tauran a exprimé ses opinions lors d'entretiens radiophoniques à la veille du Forum catholique-musulman (2008). Plus récemment, il a prononcé un discours à la Faculté de théologie de Grenade lors du congrès intitulé "Christianity, Islam and Modernity» (2010).

14. Voir : <http://www.vatican.va/holy_father/benedict_xvi/speeches/2008/november/ documents/hf_ben-xvi_spe_20081106_cath-islamic-leaders_fr.html>, consulté par le traducteur le 12 septembre 2013. 
catholiques et musulmans, le pape Benoît insiste sur l'importance d'une action concertée pour garantir la dignité humaine au milieu d'un relativisme mondialisé que Benoît voit comme une formidable menace pour l'avenir de la société humaine. Le pape Benoît affirme que chacune des traditions est capable à sa manière d'offrir des arguments contre la force corrosive du relativisme. Ainsi, il ne croit pas à la nécessité de chercher une position commune pour avancer des arguments plus convaincants. Selon lui, les deux traditions peuvent offrir chacune leurs propres arguments, s'appuyant pour cela sur leur conception particulière de Dieu, de l'homme et du monde.

Nous devrions donc œuvrer ensemble pour promouvoir le respect authentique de la dignité de la personne humaine et les droits fondamentaux de l'homme, même si nos visions anthropologiques et nos théologies les présentent de façon différente. Il y a un important et vaste domaine dans lequel nous pouvons agir ensemble pour défendre et promouvoir les valeurs morales qui font partie de notre héritage commun ${ }^{15}$.

Cette réponse, réitérée dans des allusions subséquentes à $\mathrm{CW}$, laisse entendre que la position actuelle du Vatican ne repose pas nécessairement sur une promotion active du dialogue islamo-chrétien, mais sur la reconnaissance d'une affinité fondamentale des deux traditions pour la défense des valeurs fondamentales de l'humanité. Il en ressort que des discussions de nature purement théologiques cèdent davantage la place à des questions plus pragmatiques sur une vision de l'être humain et de sa place dans le monde.

\subsection{L'Église d'Angleterre: L'éthique interreligieuse communicative du bien commun chez Rowan Williams}

Alors qu'on pourrait dire de l'approche du Vatican qu'elle est théologiquement minimaliste par rapport à sa très grande insistance sur des résultats d'ordre pragmatique, la réponse de l'archevêque de Cantorbéry, Rowan Williams, est de loin la réflexion théologique la plus poussée quant au sens à donner à l'interpellation des leaders du monde chrétien que constitue $\mathrm{CW}^{16}$.

15. Ibid.

16. Notez les interventions très médiatisées de Williams au sujet de l'islam, en particulier son discours à l'Université Al-Azhar du Caire en Égypte, <http://www.archbishopof 
Il est important de souligner l'engagement majeur de l'Église d'Angleterre auprès d'importants interlocuteurs du monde musulman avant même la publication de CW, plus particulièrement avec l'Université Al-Azhar au Caire (Haddad et Smith 2009, 375). Cette coopération entre Al-Azhar et l'Église d'Angleterre adoptait à l'origine une optique avant tout pragmatique, mais elle a depuis été supplantée par des réflexions théologiques fondamentales en réponse au cœur explicitement théologique de CW.

Monseigneur Williams propose une lecture réaliste de $\mathrm{CW}$, admettant d'emblée la futilité d'adopter des conclusions hâtives au sujet d'importants points communs entre les traditions. Malgré cette mise en garde, il reconnaît que cette lettre produit un impact immédiat sur le monde chrétien en ce qu'elle est exprimée dans un langage qui peut être compris par les chrétiens qui la lisent en toute bonne foi. Ainsi, pour Williams, la réussite de la lettre découle d'une performance linguistique. Il suggère qu'en dépit d'animosités persistantes entre chrétiens et musulmans, souvent instrumentalisées par des forces non religieuses, la publication du document est en elle-même un important acte de langage:

Nous voyons dans votre lettre l'expression d'une reconnaissance plus modeste, mais en définitive dénotant un espoir plus réaliste, que les façons dont nous comme chrétiens et comme musulmans parlons de Dieu et de l'humanité ne sont pas simplement des systèmes mutuellement inintelligibles. C'est comme si votre invitation nous disait: «trouvons une manière de reconnaître que sur certains sujets nous partageons un langage assez commun pour que nous puissions poursuivre à la fois un dialogue exploratoire et une coopération pacifique avec intégrité et sans compromettre nos croyances fondamentales. » (The Royal Aal al-Bayt Institute for Islamic Thought 2009, 95)

CW représente au bas mot la reconnaissance de l'existence au moins d'un langage commun, le point de départ essentiel pour un échange fructueux. C'est pourquoi CW est analysé selon son potentiel communicatif, le fondement nécessaire pour la réalisation d'une possible éthique partagée entre ces traditions.

Afin de clarifier davantage la question fondamentale du langage se rapportant à Dieu, Rowan Williams aborde la différence de sens que les deux traditions accordent à la révélation ainsi que les dissemblances qui en découlent dans leur appréhension des écritures. 
Bien que les deux traditions se définissent par leurs textes sacrés, les écritures ont un rôle assez différent dans le christianisme et dans l'islam. Dans la tradition chrétienne, les Écritures constituent un témoignage faisant autorité, relatant l'implication de Dieu dans l'histoire de son peuple Israël et dans l'histoire de Jésus de Nazareth, identifié par la foi chrétienne comme étant le Fils de Dieu. Dans la tradition musulmane, les Écritures n'ont pas cette fonction première de témoigner. Plutôt, le Coran se présente comme la commande de Dieu, exhortant les gens à une existence droite en parfaite soumission à sa volonté. Monseigneur Williams avance qu'il existe des manières d'arbitrer entre ces deux façons d'aborder les Écritures au bénéfice mutuel des deux parties.

L'attention portée à l'étude des Écritures fait ressortir une attitude d'ouverture au sens profond de la communication. Cela est amené par la réalisation que le fait d'être déterminé par la présence de Dieu ouvre des perspectives d'échanges respectueux avec son interlocuteur. Le potentiel communicatif d'une telle ouverture est fondé sur une compréhension particulière de Dieu dont la présence nous pousse à rejeter l'animosité et l'anxiété déraisonnable envers son prochain.

Puisqu'il est essentiel à la communication entre les deux traditions de clarifier les règles fondamentales qui gouvernent le langage qu'on applique à Dieu, Rowan Williams n'hésite pas à affronter un point de litige majeur de la communication interreligieuse entre les traditions chrétienne et musulmane. Comme le texte de CW insiste sur l'importance capitale de l'unicité de Dieu telle qu'affirmée dans le langage de la tradition musulmane, l'archevêque de Cantorbéry offre volontiers une version chrétienne de la manière selon laquelle cette unicité est affirmée par la nomenclature trinitaire. Bien que le langage trinitaire chrétien et son fondement dans l'interprétation chrétienne de la révélation divine comme trinitaire choquent assurément les oreilles musulmanes, Williams fait remarquer que si le langage trinitaire est vu comme secondaire, alors les bases mêmes de la communication entre les deux traditions demeureront dans un constant état de perturbation. Il vaut alors mieux commencer par l'exigeante tâche d'établir d'une manière compréhensible la façon dont la tradition chrétienne se comprend elle-même et communique cette compréhension d'ellemême, à la lumière de sa réception de la révélation biblique de Dieu au monde entier.

S'appuyant sur les enseignements des Pères de l'Église sur la nature trinitaire de Dieu, Monseigneur Williams tente de démontrer que ce langage n'indique en aucun cas une multiplicité de dieux (Williams, 103). Les 
chrétiens sont plutôt invités à nommer Dieu comme la Trinité du Père, Fils et Saint-Esprit. L'appellation chrétienne de Dieu comme trinitaire est en réponse à l'activité rédemptrice de Dieu dans l'histoire du peuple d'Israël et dans l'histoire de Jésus de Nazareth. Il n'y a pas trois êtres distincts l'un de l'autre, pas plus qu'il n'y a de division tripartite d'un même Dieu. Il n'y a qu'une vie divine manifeste dans l'interdépendance de l'action divine du partage de l'unique vie du Père, Fils et Saint-Esprit. La logique du partage absolu de la vie trinitaire entre Père, Fils et Saint-Esprit n'est nulle autre que la logique de l'amour. En conséquence, l'identification chrétienne de Dieu à travers une nomenclature trinitaire est la même chose que l'identification de Dieu comme amour.

Comme il a été dit plus haut, aborder la logique de l'appellation chrétienne de Dieu est nécessaire afin de dissiper, ou du moins amoindrir, les appréhensions des musulmans au sujet de ce langage, car pour ceux-ci, le langage sur Dieu est régi par le principe de tawhid, l'unicité absolue de Dieu. La discussion sur la manière de nommer Dieu permet également de favoriser un dialogue dont le point de départ avait été fixé par CW dans le double commandement de l'amour de Dieu et de l'amour du prochain. Du point de vue du langage chrétien sur Dieu, le double commandement est compris comme étant une obligation faite par Dieu en tant qu'amour, ou comme l'éternelle action de partage d'une seule vie divine, la vie du Dieu trinitaire.

Quand Dieu agit envers nous avec compassion afin de nous libérer du mal, de voir aux conséquences de notre rébellion contre lui et de nous rendre capables de faire appel à lui avec confiance, c'est là une émanation naturelle (mais non automatique) de sa propre action sans fin. L'amour désintéressé partagé, qui est la vie même de Dieu, devient réel pour notre bénéfice dans l'amour désintéressé de Jésus. Et c'est à cause de l'amour préalable de Dieu pour nous que nous devenons capables et sommes enjoints à aimer Dieu. (Williams, 106)

Cette discussion résolument chrétienne sur Dieu sert d'abord à rappeler l'importance capitale de sa prédisposition éternelle à l'amour. Le partage éternel de vie divine au sein de la vie trinitaire est manifeste dans le débordement de vie divine qui crée tout ce qui existe. Pour la tradition chrétienne, cette surabondance créatrice d'amour divin se manifeste tout particulièrement dans la volonté divine de réparer et de perfectionner la création par le don sacrificiel de vie divine inscrit dans l'histoire humaine à travers Jésus de Nazareth. La manifestation d'amour divin à travers la 
vie du Fils démontre une caractéristique importante de l'amour divin comme acte de vulnérabilité.

Les implications de la logique de l'amour sur un christianisme "performant» sont nombreuses. La logique d'action qui entraîne la réponse au double commandement est la même logique d'amour qui fait que la vie d'une personne est vulnérable dans sa relation tant à Dieu qu'à son prochain.:

Nous cherchons dans nos vies à manifester certaines des caractéristiques de l'amour même de Dieu. Nous savons que cela peut représenter un risque pour nous. D'aimer là où nous ne pouvons espérer être aimés en retour nous met dans une position de vulnérabilité, et nous pouvons oser cela seulement dans la puissance de l'Esprit-Saint de Dieu, qui crée en nous un certain écho, une certaine part de l'amour même du Christ. (Williams, 108)

L'archevêque avance que cette caractérisation de l'amour de Dieu constitue un important point de rencontre entre chrétiens et musulmans. Elle met les musulmans au défi de déceler des possibilités de rédemption dans des situations de souffrance et d'humiliation, en même temps qu'elle avertit les chrétiens de ne pas simplement s'approprier cette posture de vulnérabilité comme un prétexte à l'inaction lorsque confronté aux injustices dans le monde.

En explicitant la logique du langage trinitaire sur Dieu, Williams conclut que cette logique affirme en définitive une position partagée par les trois traditions abrahamiques au sujet de l'unité indivisible de Dieu. À ses yeux, CW représente une avancée positive pour surmonter les réserves des musulmans au sujet de l'apparente atteinte chrétienne à l'unité de Dieu, en ce que ce texte laisse entendre que les musulmans eux-mêmes n'attribuent pas aux chrétiens la croyance en plus d'un Dieu. Ce qui est clair, cependant, c'est que cette unité ne s'affirme pas de la même manière chez les deux traditions. Le fait que ces différences soient importantes ne vient pas nier le consensus fondamental, postulé par Nostra Aetate et par la réponse initiale du Vatican, en ce qui concerne la croyance partagée en l'unicité de Dieu et la centralité de sa volonté à déterminer la vie et l'avenir de sa Création.

Portant son attention sur la communauté chrétienne, Williams décrit la vie de la communauté comme une action de grâce et une dévotion perpétuelles qui découlent de la conscience de l'amour manifesté par Dieu. Dans sa vie communautaire de louanges et de dévotion à Dieu, la communauté chrétienne est profondément marquée par la réponse du peuple 
d'Israël à la sollicitude providentielle de Dieu, exprimée dans les psaumes. $\mathrm{CW}$ signale notamment le rôle primordial des psaumes dans toutes les religions abrahamiques pour exprimer leur dévotion à Dieu par une réaction dynamique à l'œuvre de Dieu.

En nous révélant certaines des richesses de la dévotion du Coran, votre lettre nous permet d'apprécier à nouveau les richesses des Psaumes. Peut-être que dans l'avenir, l'affirmation du Coran «Nous avons donné les psaumes à David» $(4: 163)$ nous encouragera ensemble à explorer plus avant nos traditions et nos pratiques de louanges, et à découvrir comment chacun à sa manière nous pouvons chercher à offrir à Dieu toute notre imagination et notre sensibilité humaine dans un acte unifié de louange. (Williams, 113-114)

Bien que les motivations qui poussent à la dévotion et à la glorification de Dieu soient différentes chez les chrétiens et chez les musulmans, chacune des traditions se consacre à l'activité continue de reconnaître la grandeur de Dieu. Les musulmans et les chrétiens sont unis par cette activité dans le témoignage rendu à la source suprême de vie en plénitude.

Pour l'archevêque de Cantorbéry, le travail solidaire des musulmans et des chrétiens se fonde sur ce qu'il identifie comme étant déterminé par une préoccupation capitale que partagent les deux traditions. Avoir conscience d'être ultimement déterminé par dessein divin sert de moyen pour voir au-delà des articulations linguistiques divergentes sur la nature de cette préoccupation capitale. Chaque tradition peut affirmer qu'une caractéristique partagée du Dieu source ultime en tant que déterminant la vie des musulmans comme des chrétiens est qu'il est hors de portée des manipulations humaines tout en suscitant une réponse commune de dévotion par le truchement de sa présence gratuite dans la vie des individus. Williams identifie cette présence gratuite de Dieu comme étant l'amour qu'il porte à sa création, décrivant la réponse humaine à la présence divine comme une indication de la réception d'un don gratuit (Williams, 98). Comme l'indiquent plusieurs réactions chrétiennes et musulmanes à $\mathrm{CW}$, l'exploration de la qualité et de la dynamique de ce don gratuit ainsi que du geste de son offrande est certainement une manière féconde d'articuler les structures profondes de cette orientation existentielle fondamentale commune aux deux traditions.

La réponse à ce don gratuit se manifeste d'abord à travers la rencontre du prochain, où l'on entend par prochain l'étranger qui est toujours présent dans une vie. D'où l'insistance à la fois dans les traditions juive et chrétienne sur le commandement d'aimer son prochain, un commandement 
que CW désigne également comme une composante fondamentale de la tradition islamique. La préoccupation capitale qui doit nous habiter, celle d'être déterminés par ce que l'on considère comme la source de la vie, est inséparable de l'orientation pratique que l'on donne à sa vie.

Le défi posé par le double commandement est qu'il unit la parfaite dévotion que l'on manifeste à Dieu aux retombées concrètes de la vie ordinaire que l'on mène, plus spécifiquement la rencontre que l'on fait avec ceux dont la présence nous est étrangère et dérangeante. L'appel à aimer son prochain est un impératif éthique qu'il est plus facile de prononcer que de réaliser. Il est particulièrement impératif que cet appel soit entendu dans le monde d'aujourd'hui, où une désinformation généralisée crée dans le monde occidental une atmosphère de peur et de suspicion envers les musulmans.

La pertinence de $\mathrm{CW}$ réside dans la volonté du texte à prendre la pleine mesure de cette situation, dans un contexte de mondialisation où musulmans et chrétiens sont devenus des prochains les uns pour les autres, c'est-à-dire qu'ils sont devenus cet autre qui peut vraiment déranger. À cet égard, les réactions souvent ouvertement violentes aux actions d'autrui illustrent clairement ce qui est en jeu actuellement dans les relations islamochrétiennes. Rowan Williams fait ressortir ce point en insistant sur l'établissement d'une relation fondée sur l'amour avec un prochain qui dérange assurément et qui peut même sembler être son ennemi ${ }^{17}$.

Du point de vue chrétien, la véritable importance du double commandement réside précisément dans cette relation au prochain, ainsi que par la réalisation active de ce commandement en triomphant des énergies violentes inhérentes à cette rencontre. Corollairement, la tradition musulmane qui se fie sur le rappel constant de la miséricorde de Dieu comporte le potentiel communicatif constructif à triompher des forces de la violence lorsqu'en contact avec la présence dérangeante du prochain de foi chrétienne. Les potentiels communicatifs positifs inhérents au commandement d'aimer son prochain, de se tourner vers son prochain en se rappelant la miséricorde de Dieu, forment par conséquent la base commune d'un engagement interconfessionnel dans l'action communicative concrète en vue du bien commun.

17. Notez l'habileté avec laquelle Williams évite la caractérisation souvent sentimentale du concept de prochain qui prévaut dans les discussions sur le double commandement (Williams, 118-119). 
L'édification d'une éthique théologique de la communication repose sur une prise de conscience mutuelle que la violence perpétrée pour défendre les convictions et les croyances profondément ancrées d'une tradition n'est rien d'autre qu'une usurpation d'une prérogative divine, une interruption du plan providentiel de Dieu pour l'humanité.

Nous pouvons alors conclure que plus nous sommes sérieux, en tant que croyants sincères, sur la nature véridique de nos convictions, plus il sera probable que nous nous détournerons de la violence au nom de la foi, et que nous serons convaincus que Dieu, le véritable réel, demeurera vrai, divin et immuable, quels que soient les échecs et les réussites de la société et de l'histoire humaines. Et nous serons conscients que d'essayer de contraindre une allégeance religieuse par la violence est en réalité une manière de tenter de remplacer la puissance divine par une puissance humaine, d'où l'insistance coranique qu'il ne peut y avoir de contrainte en matière de foi religieuse (al-Baqarah, 2:256) et l'appui dans votre lettre à la «liberté de religion». (Williams, 123)

En abordant la possibilité d'un devenir partagé entre musulmans et chrétiens, Monseigneur Williams évoque l'importance de considérer l'autre comme un prochain à la lumière d'une perspective eschatologique plus globale, qui dissout toute semblance d'inimitié par la réalité subversive de l'avènement du jugement eschatologique de Dieu.

[L'enseignement radical de Jésus sur le Jugement dernier] nous montre à accepter comme notre prochain même ceux qui s'opposent à nous. Cela est requis en partie par l'humilité devant les desseins de Dieu et par les limites de notre clairvoyance; car nous ne savons pas, comme des chrétiens l'ont souvent dit, qui parmi ceux qui luttent contre nous aujourd'hui seront nos amis au jour dernier, quand nous nous tiendrons devant notre Juge. (Williams, 120)

Cette perspective eschatologique expose également le danger que représente la construction d'identités religieuses soumises à la vision d'objectifs politiques particuliers et restreints. La force de l'éthique communicative partagée par les deux traditions monothéistes, fondée sur le double commandement, réside dans sa capacité à mettre en doute la prétendue primauté des visées politiques, ouvrant ainsi à la possibilité d'un horizon partagé.

L'éthique théologique de la communication proposée par Rowan Williams rejette en bloc la mobilisation d'identités religieuses militantes visant des fins politiques mal conçues. Par contraste avec l'infâme logique 
de la théologie politique de l'État, l'archevêque voit dans une éthique religieuse partagée une source efficace servant à contrer la logique meurtrière qui définit le monde (Williams, 125).

L'impératif éthique de promouvoir le bien commun tout autour du globe, c'est-à-dire l'engagement actif en faveur de la paix, s'appuie sur la logique des enseignements de Jésus concernant l'amour du prochain. Une dimension importante de cette logique consiste en une prise de conscience critique qui mène à la réalisation qu'il faut aimer son prochain, non pas par une tentative de gommer les différences sur un hypothétique terrain neutre, mais par l'acceptation radicale de la présence continue de ces différences. On ne doit jamais simplement passer outre les différences ni les relativiser, mais les considérer du point de vue plus large de la transcendance absolue du divin. Les positions particulières au sujet de la vérité, bien qu'elles visent à être des expressions authentiques de la réception de la révélation de l'absolu, ne doivent pas devenir des sources pour une théologie politique associée à des objectifs spécifiques et limités de ce monde.

Le pouvoir du double commandement tel que mis de l'avant par CW comme terrain d'entente entre christianisme et islam est compris par l'archevêque de Cantorbéry comme un appel à interpréter ensemble une théologie politique critique partagée des traditions abrahamiques. Cette théologie politique se définit par son refus de légitimer un pouvoir politique ainsi que par sa critique détaillée de conceptions purement laïques du bien commun, consciente des restrictions que celles-ci imposent sur les multiples visages de l'épanouissement humain dans une société pluraliste.

\section{Conclusion}

En contemplant la possibilité d'une théologie politique partagée entre l'islam et le christianisme, l'archevêque de Cantorbéry place à l'avant-plan ce qui pourrait être une dimension tacite de CW. En fait, le seul point de convergence entre les deux types de réponses européennes discutés plus haut se trouve précisément dans leur reconnaissance de l'importance grandissante de la théologie politique islamique. Toutefois, ce point de convergence révèle de profondes différences entre les deux réactions à cette théologie politique islamique, réactions déterminées par des visions alternatives de l'identité religieuse européenne. Celle du pape Benoît peut être décrite comme une approche quérulente, marquée par l'accent renouvelé qu'il met sur l'articulation des universels chrétiens en recourant au concept patristique de vera religio. C'est ce dernier concept qui au bout du compte 
détermine l'idée que se fait le pape Benoît du prochain. Par contraste, Rowan Williams - par une analyse de l'identité du prochain d'après la logique trinitaire de la conception chrétienne de Dieu — arrive à l'affirmation d'une éthique abrahamique partagée d'un bien commun reposant sur de solides bases transcendantes. Selon la conception de Williams d'une éthique abrahamique partagée, la vera religio concerne moins la confirmation d'universels issus d'une tradition particulière. Elle est plutôt conçue comme la vérité de la synergie des religions monothéistes qui triomphent de la logique de violence produite par les conceptions du bien commun marquées par l'oubli du transcendant. C'est pour cette raison qu'il reconnaît l'importance aujourd'hui de la théologie politique islamique dans sa persistance à rappeler au monde le besoin de retourner à sa source première d'épanouissement.

\section{Références}

Baum, G. (2009), The Theology of Tariq Ramadan: A Catholic Perspective, South Bend, University of Notre Dame Press.

Collectif (2007), "Loving God and Neighbor Together: A Christian Response to A Common Word Between Us and You ", New York Times, 11 novembre.

Haddad, Y. Y. et Smith, J. I. (2009) "The Quest for "A Common Word": Initial Christian Responses to a Muslim Initiative ", Islam and ChristianMuslim Relations, 20/4, p. 369-388.

Helmick, R, (2001a), "Does Religion Fuel or Heal in Conflict?", R. Helmick et R. Peterson, dir., Forgiveness and Reconciliation: Religion, Public Policy, and Conflict Transformation, Philadelphia, Templeton Foundation Press.

Huntington, S. (1996), The Clash of Civilizations and the Remaking of World Order, New York, Simon and Schuster.

Lewis, B. (1990), "The Roots of Muslim Rage», The Atlantic.

Lumbard, J. (2009), "The Uncommonality of "A Common Word” ", Crown Paper, 3, Brandeis University, Crown Center for Middle East Studies.

Madigan, D., s.j. (2008), «A Common Word Between Us and You: Some Initial Reflections », disponible sur <http://www.thinkingfaith.org/articles/ 20080118_9.htm>, consulté le 09 décembre 2013. 
Migliore. C., Monseigneur (2008), Catholicism and Islam: Points of Convergence and Divergence, Encounter and Cooperation, University of Notre Dame (The Nanovic Institute for European Studies).

Ramadan, T. (2006), "A Struggle Over Europe's Religious Identity ", New York Times, 20 septembre.

Salvatore, A. (2007), The Public Sphere Liberal Modernity, Catholicism, Islam, New York, Palgrave.

Speelman, G. M. (2010), "The Background of "A Common Word" ", Exchange, 39, p. 109-120.

Open Letter to His Holiness, <http://ammanmessage.com/media/openLetter/ english.pdf>.

Tauran, J. L., Cardinal (2008), «Interreligious Dialogue. A Risk or an Opportunity?", Thinking Faith The Online Journal of the British Jesuits, 31 mai, <http://www.thinkingfaith.org/articles/20080531_1. htm>, consulté le 15 novembre 2010.

The Royal Aal al-Bayt Institute for Islamic Thought (2009), "Response by the Archbishop of Canterbury in A Common Word Between Us and You, <http://www.acommonword.com/category/newfruits/publications>.

Williams, R. (2004), "Speech at Al-Azhar University in Cairo, Egypt », disponible sur <http://rowanwilliams.archbishopofcanterbury.org/articles. php/1299/archbishops-address-at-al-azhar-al-sharif-cairo>, consulté le 09 décembre 2013.

(2008), "Civil and Religious Law in England: A Religious Perspective in the UK», disponible sur <http://rowanwilliams.arch bishopofcanterbury.org/articles.php/1137/archbishops-lecture-civil-andreligious-law-in-england-a-religious-perspective>, consulté le 09 décembre 2013.

\section{Résumé}

Cet article propose une évaluation critique des deux réponses d'envergure à «A Common Word» originaire de centres traditionnels de la chrétienté européenne. Dans les deux cas, la notion de bien commun joue un rôle central dans la détermination de la forme et de l'orientation de la coopération interreligieuse dans un contexte mondial. Alors que les réponses émanant du Vatican sont lues comme une expression de la pragmatique interreligieuse, la seconde, un engagement plus solide de Rowan Williams est pré- 
senté comme une tentative de développer une éthique interreligieuse de la communication. L'examen critique de ces deux visions de la solidarité interreligieuse conduit à des conclusions significatives quant à la notion d'autrui, une catégorie centrale partagée par toutes les traditions abrahamiques.

\section{Abstract}

This paper offers a critical evaluation of two prominent responses to "A Common Word" hailing from traditional centers of European Christianity. In both, the concept of the common good plays a prominent role in determining the shape and direction of interreligious cooperation in a global context. While the responses emanating from the Vatican are read as an expression of interreligious pragmatics, the second, more robust engagement of Rowan Williams is presented as an attempt to develop an interreligious communicative ethic. The critical examination of these two visions of interreligious solidarity leads to significant conclusions regarding the concept of the neighbour, a central category shared by all Abrahamic religious traditions. 